\title{
A chronicle of single-crystal neutron diffraction at Oak Ridge
}

\author{
Bryan C. Chakoumakos
}

Neutron Scattering Division, Oak Ridge National Laboratory, Oak Ridge, Tennessee 37831 chakoumakobc@ornl.gov

Signature applications and features of single-crystal neutron diffraction are high resolution nuclear structure analysis, magnetic structure and spin density determinations, isotopic contrast variation (particularly $\mathrm{D}_{2} \mathrm{O} / \mathrm{H}_{2} \mathrm{O}$ ) for nuclear structural studies, lack of radiation damage when using crystals of biological molecules such as proteins, and the fidelity to measure nuclear and magnetic diffuse scattering with elastic discrimination. Neutron scattering lengths have no fall off with scattering angle, so neutron diffraction offers a greater opportunity to accurately determine atomic displacement parameters. The markedly different neutron scattering powers of the elements as compared to X-ray scattering often give much better discrimination of solid solutions involving neighboring elements, e.g., $\mathrm{Mn}$ and Fe. Examples of these useful features of neutrons will be given from magnetic systems and energy materials. The emergence of neutron scattering science at Oak Ridge proceeded from a succession of three important research reactors, Graphite, ORR, and HFIR, followed by the world's most powerful spallation source, SNS. The nascent suite of single-crystal neutron diffractometers now at HFIR and SNS is unparalleled worldwide and offers the potential to re-assert single-crystal diffraction using neutrons as a significant tool to study nuclear and magnetic structures of small unit cell crystals, nuclear structures of macromolecules, and diffuse scattering. 